\title{
Comparison of the Time Domain Windows Specified in the ISO 18431 Standards Used to Estimate Modal Parameters in Steel Plates
}

\author{
Jhonatan Camacho-Navarro, ${ }^{1}$ R. Guzmán-López, ${ }^{2}$ Sergio Gómez, ${ }^{2}$ and Marco Flórez ${ }^{1}$ \\ ${ }^{1}$ Escuela de Ingeniería Electrónica, Universitaria de Investigación (UDI), Grupo de Investigación en Procesamiento de Señales (GPS), \\ Bucaramanga, Colombia \\ ${ }^{2}$ Universidad Pontificia Bolivariana, Bucaramanga, Colombia
}

Correspondence should be addressed to Jhonatan Camacho-Navarro; camacho.navarro.jhonatan@gmail.com

Received 29 April 2016; Revised 14 July 2016; Accepted 3 August 2016

Academic Editor: Massimo Viscardi

Copyright (C) 2016 Jhonatan Camacho-Navarro et al. This is an open access article distributed under the Creative Commons Attribution License, which permits unrestricted use, distribution, and reproduction in any medium, provided the original work is properly cited.

\begin{abstract}
The procedures used to estimate structural modal parameters as natural frequency, damping ratios, and mode shapes are generally based on frequency methods. However, methods of time-frequency analysis are highly sensible to the parameters used to calculate the discrete Fourier transform: windowing, resolution, and preprocessing. Thus, the uncertainty of the modal parameters is increased if a proper parameter selection is not considered. In this work, the influence of three different time domain windows functions (Hanning, flat-top, and rectangular) used to estimate modal parameters are discussed in the framework of ISO 18431 standard. Experimental results are conducted over an AISI 1020 steel plate, which is excited by means of a hammer element. Vibration response is acquired by using acceleration records according to the ISO 7626-5 reference guides. The results are compared with a theoretical method and it is obtained that the flat-top window is the best function for experimental modal analysis.
\end{abstract}

\section{Introduction}

Physical behavior of complex engineering systems can be studied through prediction and simulation analysis by means of specialized software $[1,2]$. Thus, it is possible to check abnormal performance with the help of monitoring methods based on simulation tools. In particular, the analysis of structural dynamics can be addressed by determining modal parameters defined by mode shapes, natural frequencies, and damping ratios. In this sense, by means of Operational Modal Analysis it is possible to conduct nondestructive testing, fatigue analysis, and issues concerned with field in structural analysis [3]. These tasks also involve updated finite element models used to predict the dynamic behavior of the structure reliability [4]. For instance, an application of modal analysis is the assessment of highway-bridge by dynamic testing and finite-element model updating [5]. Also, damage detection has been carried out in reinforced concrete beams by using modal flexibility residuals [6].
Because of the importance of modal analysis in the field of structural analysis, well established procedures to obtain proper estimations of modal parameters are required. Although there exists a huge documentation about methods used for modal analysis $[7,8]$, their practical implementation is still difficult because there are many parameters involved in the procedure, which must be decided by engineers of different areas and knowledge. In this sense, the 7626 standard ISO specifies a guideline of methodological steps to conduct experiments in order to obtain the frequency response measurement and the 18431 ISO describes the procedures for time-frequency analysis of vibrational records. Thus, by considering the recommendations of the ISO standards, the further estimation of modal model parameters by means of well-known modal analysis methods (as, e.g., peak-picking or least squares among others) is facilitated.

In this paper, a practical implementation of the abovementioned ISO standards with a special emphasis on computing the natural frequency values is demonstrated. Thus, the 
influence of using three domain window functions (Hanning, flat-top, and rectangular) to estimate natural frequencies is discussed. Experimental results are conducted over an AISI 1020 steel plate, which is excited by means of a hammer element.

\section{Theoretical Framework}

The procedure used in this paper to estimate the natural frequencies of a modal model is based on the analysis of measurements from Frequency response functions (FRFs). In this sense, the extraction of relevant frequency information is performed by applying spectrum estimation techniques to structural vibrational records. Thus, FRFs are approximated by the cross-power spectral density (PSD) between the vibrational responses.

In this section, the conceptual issues involved in the estimation of structural natural frequencies by means of FRFs are detailed. Also, fundamentals of methods used to estimate the frequency decomposition are presented, focusing on given details about the parameters with great influence for its implementation.

2.1. Frequency Response Functions (FRFs). Dynamical model of structures is constructed on physical knowledge and fundamental laws of motion according to [9]

$$
M \ddot{\mathbf{X}}+C \dot{\mathbf{X}}+K \mathbf{X}=F,
$$

where $K, C$, and $M$ are the stiffness, damping, and mass matrices, respectively. Likewise, $F(t)$ and $X(t)$ denote the forcing vector and displacement. Assuming that $F(t)$ is a delta-correlated exciting force and using properties demonstrated in the Natural Excitation Technique (NExT), it is possible to write the law motion of (1) in the form of [9]

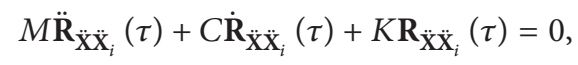

where $\mathbf{R}_{\ddot{\mathbf{X}}} \ddot{\mathbf{X}}_{i}$ is a vector of correlation functions for all positive lags $\tau>0$, between the displacement vector and a reference signal, which must be uncorrelated with respect to excitation signal. Thus, (2) establishes that correlation function between acceleration records and a reference signal can be treated as free vibration data, which allows determining modal characteristics of the structures.

Moreover to law motion and NExT equations used in methodologies for modal parameter identification, the FRF relationship in structures must be specified. The FRF for one $n$-degree of freedom system represented by (1) or (2) can be written in the form of partial fractions as is expressed in (3) (classical pole/residue) $[10,11]$.

$$
\begin{aligned}
& {\left[G_{y y}(\omega)\right]} \\
& =\sum_{k=1}^{m} \frac{\left[A_{k}\right]}{j \omega-\lambda_{k}}+\frac{\left[A_{k}\right]^{*}}{j \omega-\lambda_{k}{ }^{*}}+\frac{\left[B_{k}\right]}{-j \omega-\lambda_{k}}+\frac{\left[B_{k}\right]^{*}}{-j \omega-\lambda_{k}{ }^{*}},
\end{aligned}
$$

where $G_{y y}(\omega)$ is the output PSD matrix, $m$ is the total number of modes of interest, $\lambda_{k}=-\sigma_{k}+j \omega_{d k}$ is the pole of

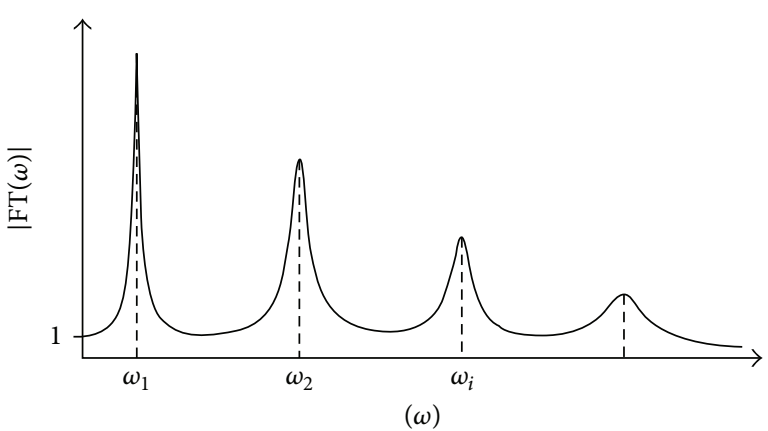

FIGURE 1: Typical FRF of an $n$-degree of freedom system.

the $k$ th mode, $\sigma_{k}$ is the modal damping (constant decay), $\omega_{d k}$ is the damped natural frequency, and the $*$ superscript denotes complex conjugate. The typical PSD curve for the FRF determined by (3) is depicted in Figure 1.

According to Figure 1, the modes $\left(\omega_{k}\right)$ located at the peak can be identified from spectral density through common signal processing as discrete Fourier transform.

2.2. Frequency Domain Decomposition (FDD). Classical approach used to estimate modal parameters is often referred to as Peak-Peaking (PP), which is a nonparametric method essentially developed in frequency domain. The main advantages of this method are its user-friendliness, simple use, and fast results obtaining. In this method, average normalized power spectral densities and frequency response functions between all the measurement points of the structure are evaluated [12]. As a result, first the natural frequencies are simply taken from the observation of the peaks on the graphs of the magnitude of the response (Figure 1). Next, damping ratios $\left(\sigma_{k}\right)$ are calculated from the sharpness of the peaks obtained by half power band method. Then, the mode shapes are calculated from the ratios of the peak amplitudes at various points in the structure [13]. Finally, modal participation factors are computed to scaled mode shapes by using measures of force exciting. In order to apply the PP method, its procedure can be summarized as follows:

(i) Determine the natural frequencies by means of the PSD computed from acceleration records by identifying all frequencies present at peaks $\left(\omega_{r}\right)$.

(ii) Estimate damping ratios $(\xi)$ by means of the loss factor $\left(n_{r}\right)$ which depends of the half power band frequencies $\left(\omega_{a}, \omega_{b}\right)$ as is illustrated in Figure 2.

According to Figure 2, the half power band comprises the frequencies where the PSD amplitude decays by $3 \mathrm{~dB}$ with respect to its maximum value. At once the half power band is determined; the loss and damping factors are computed by using

$$
\begin{aligned}
& n_{r}=\frac{\omega_{b}-\omega_{a}}{\omega_{r}} ; \\
& \xi=\frac{\omega_{b}-\omega_{a}}{2 \omega_{r}}=\frac{n_{r}}{2} .
\end{aligned}
$$




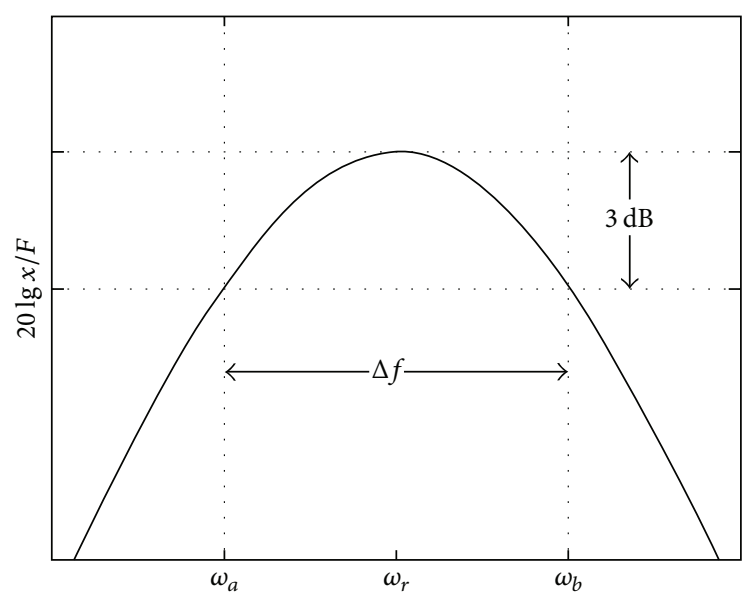

FIGURE 2: Half power band frequencies.

In this paper an enhanced method known as frequency domain decomposition (FDD) is used instead, which is an extension of PP method. It consists of three main steps [14]:

(i) Estimate the power spectral density matrix at discrete frequencies.

(ii) Do a singular value decomposition of the power spectral density.

(iii) For an $n$-degree of freedom system, then pick the $n$ dominating peaks in the power spectral density. These peaks correspond to the mode shapes.

FDD technique removes disadvantages associated with classical PP method as, for example, the difficulty to detect close modes and bias estimation. Furthermore FDD reduces the uncertainty or hardness (even impossible) of damping estimation. Also, it keeps the user-friendliness giving a feeling of the data it is dealing with.

2.3. Spectrum Estimation. Frequency response functions between input and output are approximated as cross-power spectral densities between responses while the impulse response functions are approximated by cross-correlations between responses. Cross-PSDs are obtained using Welch method (FFT based method) [15]. Welch's periodogram averaging with overlapping is a method which introduces improved properties based on basic periodogram methods:

(i) Simple Periodogram. Quotient of the squared magnitude of the Fourier transform of the signal and length of the signal.

(ii) Modified Periodogram. Certain window other than rectangular window that is applied to the signal before taking the Fourier transform. Windows solve the leakage problem.

(iii) Bartlett Periodogram Averaging. Averaging of the different blocks of the signal, which decrease variance of the signal at the expense of resolution.

Thus, the cross-power spectra between $X(n)$ and some $Y(n)$ discrete signal, computed through Welch's method, are expressed by (5). Therefore, the cross-PSD $S_{X Y}$ can be defined as the Fourier Transform of the cross-correlation function $R_{X Y}(\tau)$.

$$
\begin{aligned}
S_{X Y}= & \frac{1}{K} \sum_{k=1}^{K} S_{X Y}(k), \\
& \quad \text { where } S_{X Y}(k)=\frac{1}{N} \sum_{n=0}^{L-1}\left|w(n) R_{X Y}(\tau)^{(k)} e^{-j \omega n}\right|^{2}
\end{aligned}
$$

According to (5), the Welch method divides the signal into $k$ blocks and then increases the averaging by taking overlaps of the blocks. Thus, the $L$ samples of $R_{X Y}$ are divided into $K$ overlapping sections with $N$ samples in each block, each of which is then windowed by the WINDOW $w(n)$. Finally, it averages the periodograms of the overlapping sections to form $S_{X Y}$, the power spectral density estimation of $R_{X Y}$.

2.4. Windowing Effect. In estimating power spectral density (PSD) of a signal, there are two tradeoffs. One is frequency resolution and the other is noise in the signal. To obtain a good estimation of PSD, we should have large length of the signal but during measurements we have finite length of signal. If we take small block size, bad frequency resolution could introduce leakage in the spectrum. To reduce leakage, signals are windowed, that is, multiplied with a window in time domain. Many windows are available, each one having specific application in signal processing $[16,17]$. Figure 3 illustrates the spectral characteristics of time windows recommended by the ISO standard: Hanning, flat-top, and rectangular.

According to Figure 3, the frequency estimation for modal parameter identification is limited by the frequency resolution of the spectral density. Resolution refers to the ability of distinguishing narrowband spectral components. In this sense, the spectrum of the ideal window with high frequency resolution and good noise suppression is narrower than the main lobe width and lesser than the side lobe level. Consequently, in order to select the suitable window function, a compromise between noise suppression and resolution is required. In this sense, the flat-top and rectangular windows produce the worst/best leakage factors $(96.79 \%-$ 9.14\%), respectively, which corresponds to low and high relative side lobe attenuations. Also, it is noted that Hanning window offers balanced characteristics.

2.5. The ISO Standards. The 7626-5 and 18431 ISO standards give recommendations about recording protocol and selection of window function in order to estimate modal parameters [18]. Specifically, in the document entitled "Vibration and Shock Experimental Determination of Mechanical Mobility Part 5," it is found that the guidelines for measurements using impact excitation with an exciter which are not attached to the structure. Thus, the ISO 7626-5:1994 specifies procedures to obtain acceleration records using impact excitation. Also, signals analysis based on the discrete Fourier transform is covered where a recommendation about Hanning window 

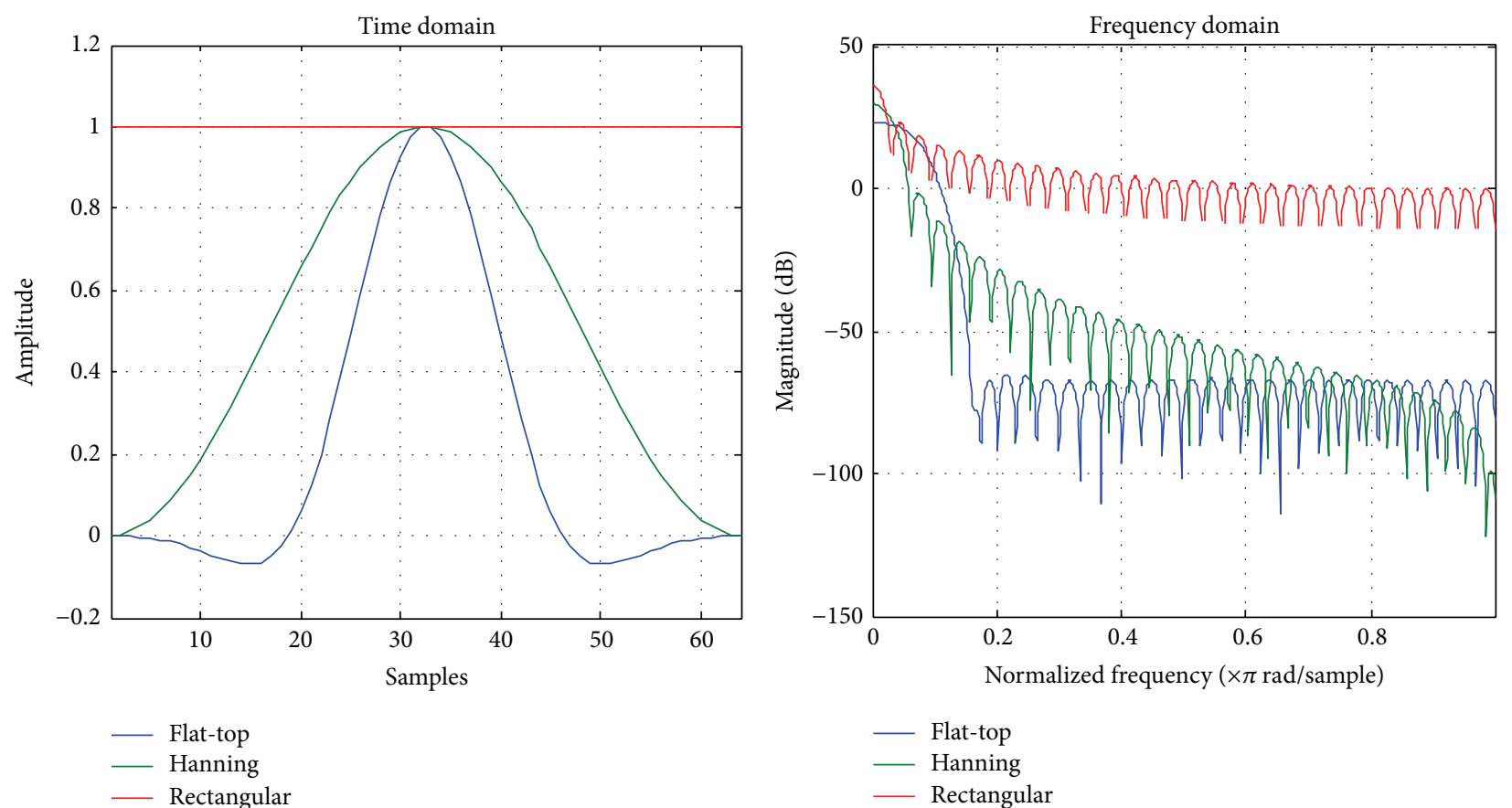

FIGURE 3: Spectral characteristics of recommended ISO-time windows.

can be justified. Similarly, the document "Mechanical Vibration and Shock Signal Processing Part 2: Time Domain Windows for Fourier Transform Analysis" which contains the ISO 18431-2:2004 standard, describes three time domain windows consisting of Hanning, flat-top, and rectangular that are suggested to be used for preprocessing samples vibration.

Considering the selected set of time windows specified in the ISO standards, in this paper the results of applying Hanning, flat-top, and rectangular windows for modal modes estimation are discussed.

\section{Results and Analysis}

The next sections show the results obtained by applying experimental and theoretical procedures. For experimental analysis the three time windows specified in the ISO standard are considered: Hanning, flat-top, and rectangular functions, while the theoretical approach is based on finite element method.

3.1. Experimental Setup. A steel plate was used in order to analyze the influence in the estimation of natural frequencies when different window functions are considered. In Figure 4, a scheme of the structure is shown.

Measurements from acceleration response were conducted over 4 points of the structure (P6, P7, P8, and P12). Vibration data were recorded under impulse hammer excitation type with sample time $T_{s}=20 \mu$ s. Then, 25.000 samples for each accelerometer were processed which correspond to 0.5 seconds of data. Figure 5 shows the recorded signals.

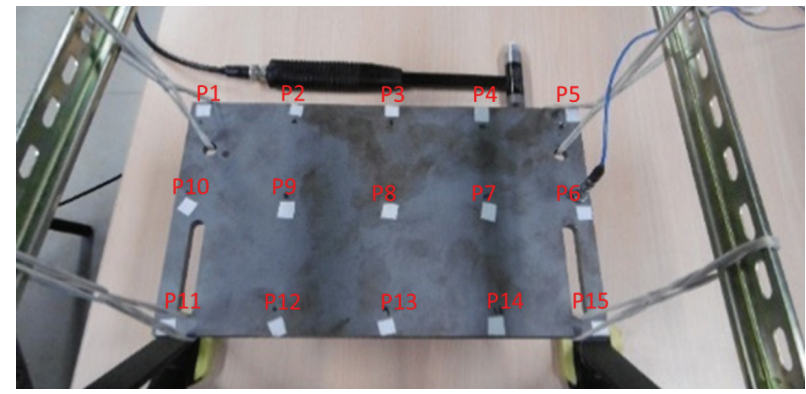

FIgURE 4: Bench structure.

3.2. Numerical Analysis. In order to calculate modal frequency of different deformation modes, finite element simulation through a numerical model was performed by using ANSYS software. The model includes the plate detailed geometry and it is characterized by density $=7733.75 \mathrm{Kg} / \mathrm{m}^{3}$, $E=200.000 \mathrm{Mpa}$, and Poison $=0.29$ parameters. The used finite elements mesh is constituted by total number of 40052 reduced integration hexahedral elements of eight integration nodes C3D8R (see Figure 6).

The modal shapes studied by means of the simulation software are depicted in Figure 7.

3.3. Experimental Results. In order to evaluate the influence of windowing effect, experimental measurements of acceleration records were processed considering windows described in previous sections. As a first result, the $2 \mathrm{Khz}$ range of interest for cross-power spectral density of the acceleration 

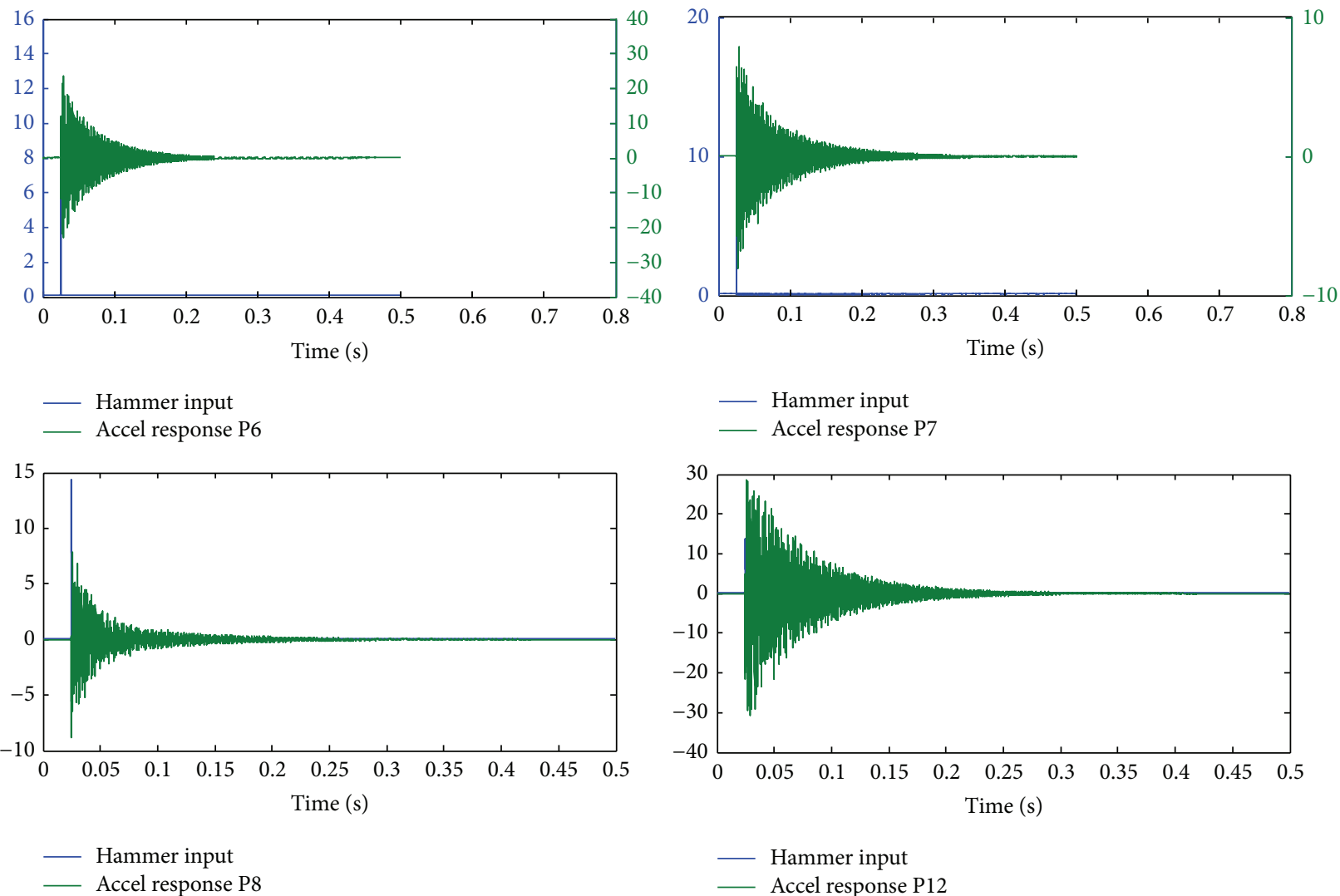

FIGURE 5: Recorded vibration signals.

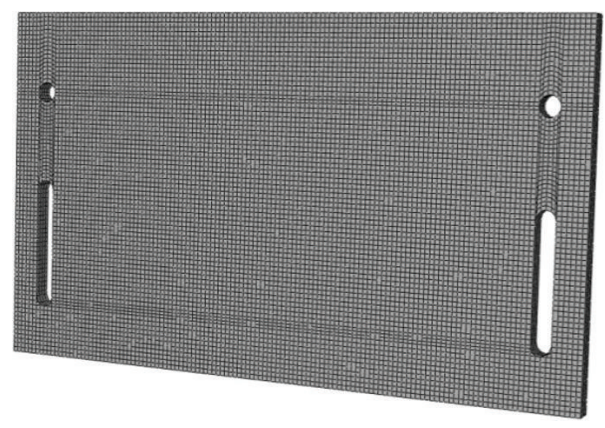

FIGURE 6: Structural finite element model.

measurements is depicted in Figure 8. The signals were divided into eight sections with $50 \%$ overlap, each section was windowed with a Hamming window, and eight modified periodograms were computed and averaged.

Also, the frequency response function computed by means of frequency domain decomposition after processing the CPSD of data in Figure 8 is depicted in Figure 9, where the amplitude in $\mathrm{db}$ corresponds to the first singular values of the PSD matrix estimated by using Hanning window function.

A comparison of the modes estimated according to ISO $7626-5$ by using the three selected time window functions is presented in Table 1.
TABLE 1: Natural frequencies $[\mathrm{Hz}]$ estimated by using three different windows.

\begin{tabular}{lcccc}
\hline $\begin{array}{l}\text { Vibrational } \\
\text { mode }\end{array}$ & $\begin{array}{c}\text { Numerical } \\
\text { model }\end{array}$ & Hanning & Flat TOP & Rectangular \\
\hline First bending & 636 & 640.9 & 634.8 & 636.3 \\
$\begin{array}{l}\text { First torsion } \\
\begin{array}{l}\text { Second } \\
\text { torsion }\end{array}\end{array}$ & 671 & 671.4 & 671.4 & 668.3 \\
$\begin{array}{l}\text { Second } \\
\text { bending }\end{array}$ & 1472 & 1465 & 1469 & 1468 \\
\hline
\end{tabular}

TAble 2: Percentage error (\%) of mode estimation with respect to numerical model.

\begin{tabular}{lccc}
\hline Vibrational mode & Hanning & Flat-top & Rectangular \\
\hline First bending & 0.7704 & 0.1887 & 0.0472 \\
First torsion & 0.0596 & 0.0596 & 0.4024 \\
Second torsion & 0.4755 & 0.2038 & 0.2717 \\
Second bending & 3.4756 & 3.1098 & 3.2927 \\
\hline
\end{tabular}

Finally, the percentage errors for each natural frequency with respect to theoretical numerical model are summarized in Table 2.

According to results in Table 2, the high error corresponds to the second bending mode, while the other modes maintain 


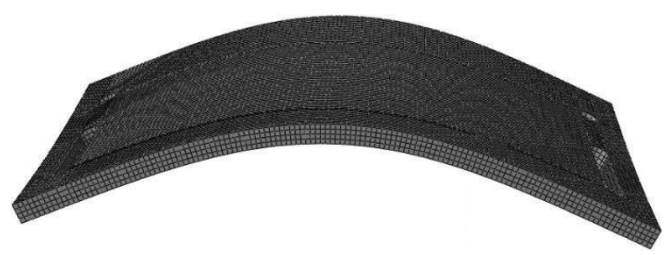

(a) First bending: $636 \mathrm{~Hz}$

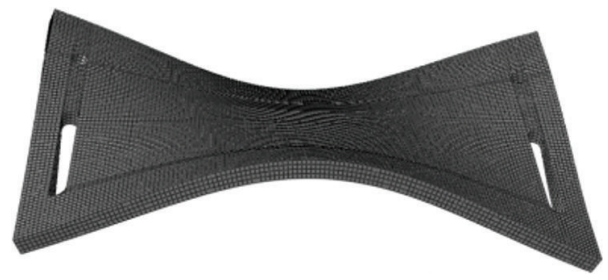

(c) Second torsion: $1472 \mathrm{~Hz}$

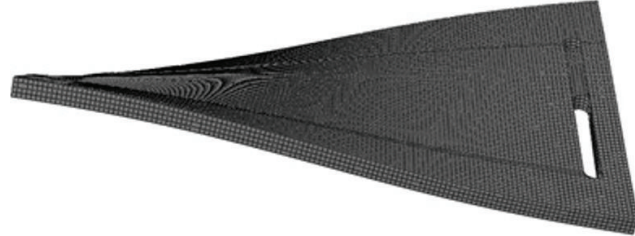

(b) First torsion: $671 \mathrm{~Hz}$

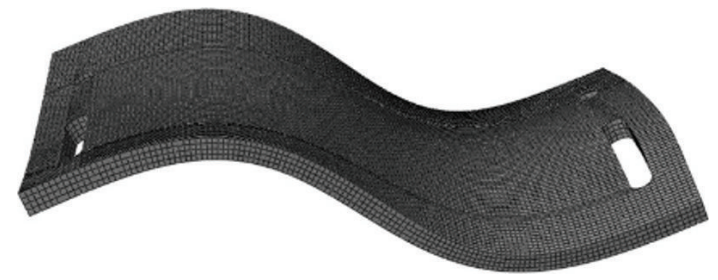

(d) Second bending: $1640 \mathrm{~Hz}$

FIGURE 7: Modal frequency obtained by numerical analysis.
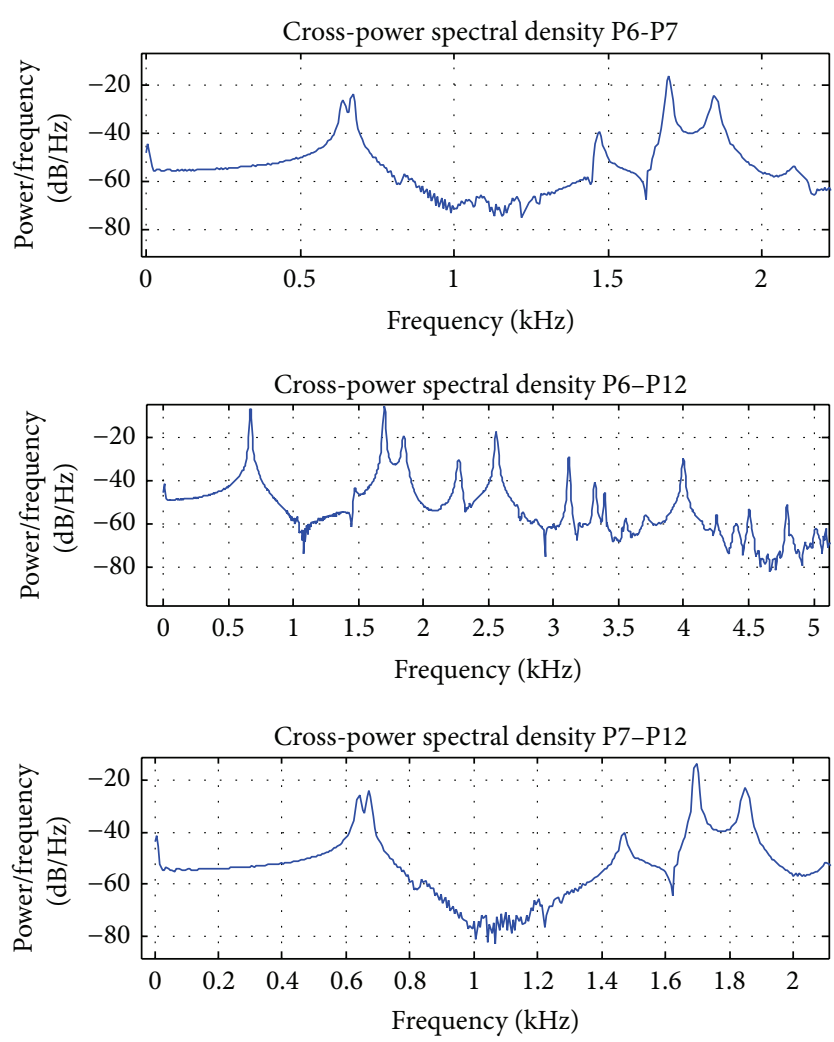
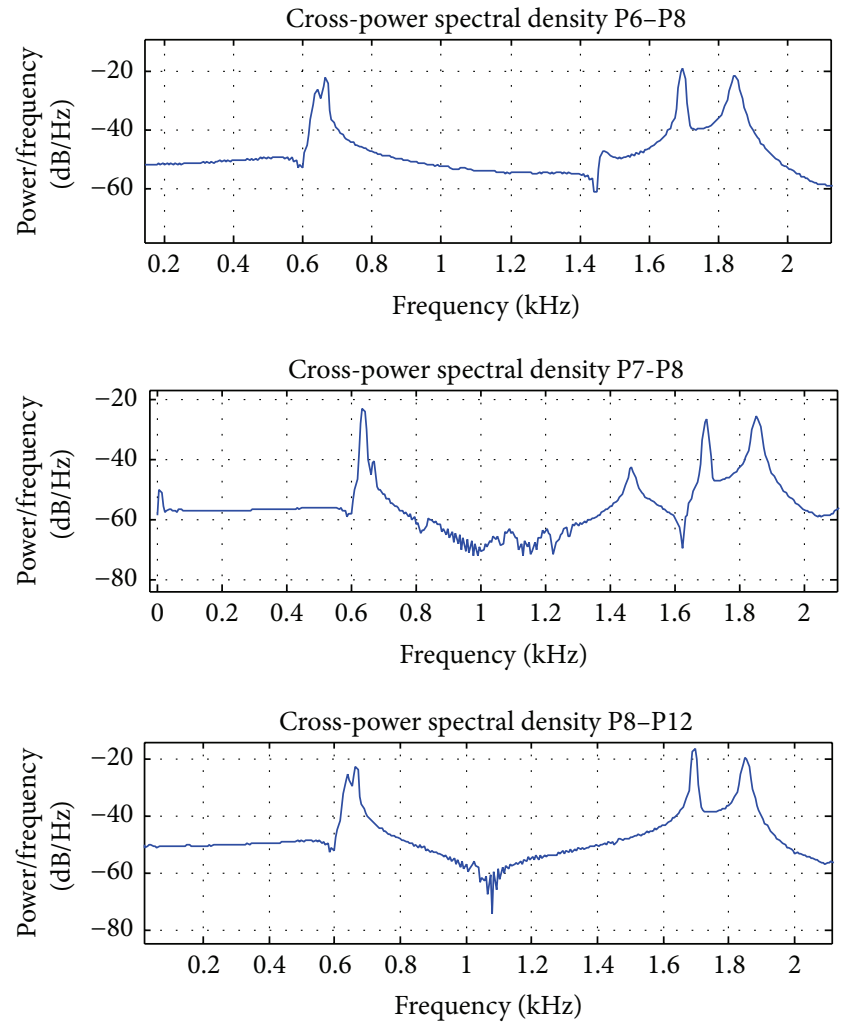

FIGURE 8: CPSD for acceleration records.

comparable values to the theoretical ones with errors lower than $1 \%$.

\section{Conclusion}

Although no meaning differences were found when the three windows specified in the ISO standard were used to estimate natural frequencies, a slight better result is obtained for flat-top function. This implies that for modal parameter estimation purposes the selection of time windowing function has low influence, with major errors for the highest modes. However, the influence of the windowing preprocessing for the analysis of different modal parameters as shape mode and factor participation should be studied. Also, further analysis should be conducted with respect to additional parameters involved in the spectrum estimation such as overlap, FFT length, and segmentation. Moreover, it is recommended to include uncertainty analysis to evaluate the influence of using different time windows. 


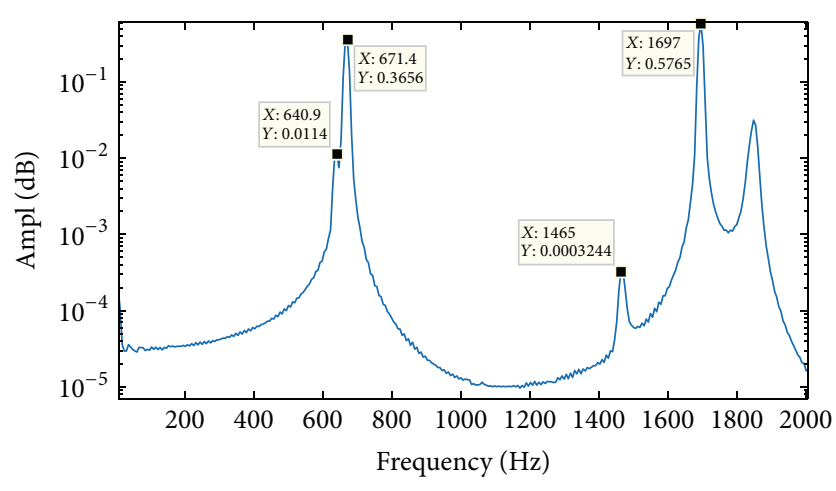

FIGURE 9: Frequency response function.

\section{Competing Interests}

The authors declare that they have no competing interests.

\section{Acknowledgments}

This work has been developed as part of a collaborative work between researches from Universitaria de Investigación y Desarrollo (UDI) and Universidad Pontificia Bolivariana (UPB), Bucaramanga, Colombia.

\section{References}

[1] E. Madenci and I. Guven, The Finite Element Method and Applications in Engineering Using $A N S Y S^{\circledR}$, Springer, Berlin, Germany, 2015.

[2] H. H. Lee, Finite Element Simulations with ANSYS Workbench 16, SDC Publications, 2015.

[3] R. Brincker and C. Ventura, Introduction to Operational Modal Analysis, John Wiley \& Sons, New York, NY, USA, 2015.

[4] M. Imregun, W. J. Visser, and D. J. Ewins, "Finite element model updating using frequency response function data: I. Theory and initial investigation," Mechanical Systems and Signal Processing, vol. 9, no. 2, pp. 187-202, 1995.

[5] J. M. W. Brownjohn, P. Moyo, P. Omenzetter, and Y. Lu, "Assessment of highway bridge upgrading by dynamic testing and finite-element model updating," Journal of Bridge Engineering, vol. 8, no. 3, pp. 162-172, 2003.

[6] B. Jaishi and W.-X. Ren, "Damage detection by finite element model updating using modal flexibility residual," Journal of Sound and Vibration, vol. 290, no. 1-2, pp. 369-387, 2006.

[7] D. J. Ewins, Modal Testing: Theory and Practice, vol. 6, Research Studies Press, Letchworth, UK, 1995.

[8] N. M. M. Maia and J. M. M. e Silva, Eds., Theoretical and Experimental Modal Analysis, Research Studies Press, Taunton, UK, 1997.

[9] J. M. Caicedo, Two structural health monitoring strategies based on global acceleration responses: development, implementation, and verification [M.S. thesis], Washington University, 2001.

[10] S. Gade, N. B. Møller, H. Herlufsen, and H. KonstantinHansen, "Frequency domain techniques for operational modal analysis," in Proceedings of the 1st International Operational Modal Analysis Conference, pp. 261-271, St. Louis, Mo, USA, 2005.
[11] R. Brincker, L. Zhang, and P. Andersen, "Modal identification from ambient responses using frequency domain decomposition," in Proceedings of the 18th International Modal Analysis Conference (IMAC'00), San Antonio, Tex, USA, February 2000.

[12] A. J. Felber, Development of a hybrid bridge evaluation system [Ph.D. thesis], University of British Columbia, 1993.

[13] N. M. M. Maia, Extraction of valid modal properties from measured data in structural vibrations [Ph.D. thesis], Imperial College London, University of London, 1988.

[14] R. Brincker, L. Zhang, and P. Andersen, "Modal identification of output-only systems using frequency domain decomposition," Smart Materials and Structures, vol. 10, no. 3, pp. 441-445, 2001.

[15] S. M. Kay, Modern Spectral Estimation, Prentice Hall, Upper Saddle River, NJ, USA, 1988.

[16] A. V. Oppenheim, R. W. Schafer, and J. R. Buck, Discrete-Time Signal Processing, vol. 2, Prentice-Hall, Englewood Cliffs, NJ, USA, 1989.

[17] C. Michael and A. F. Audrey, "The fundamentals of FFT-based signal analysis and measurement," Strategies for Choosing Windows, National Instruments Application Notes 041.

[18] S. B. Blaeser and P. D. Schomer, "Acoustical standards news," The Journal of the Acoustical Society of America, vol. 136, no. 1, pp. 439-448, 2014. 


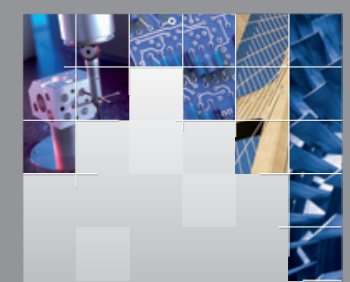

\section{Enfincering}
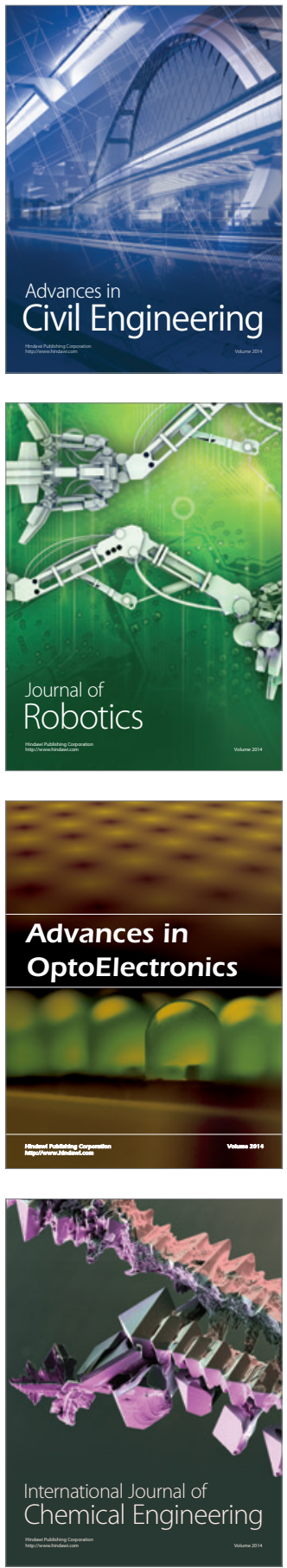

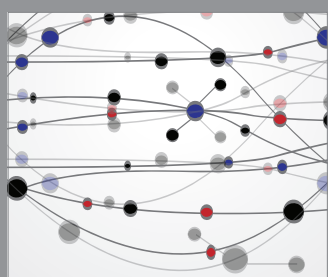

The Scientific World Journal

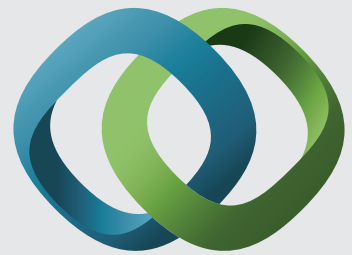

\section{Hindawi}

Submit your manuscripts at

http://www.hindawi.com
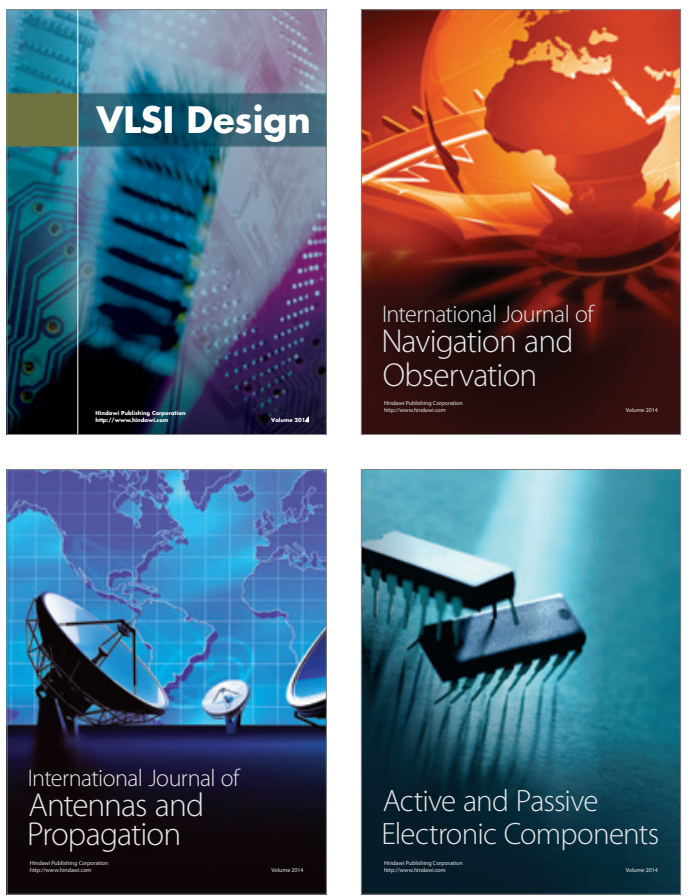
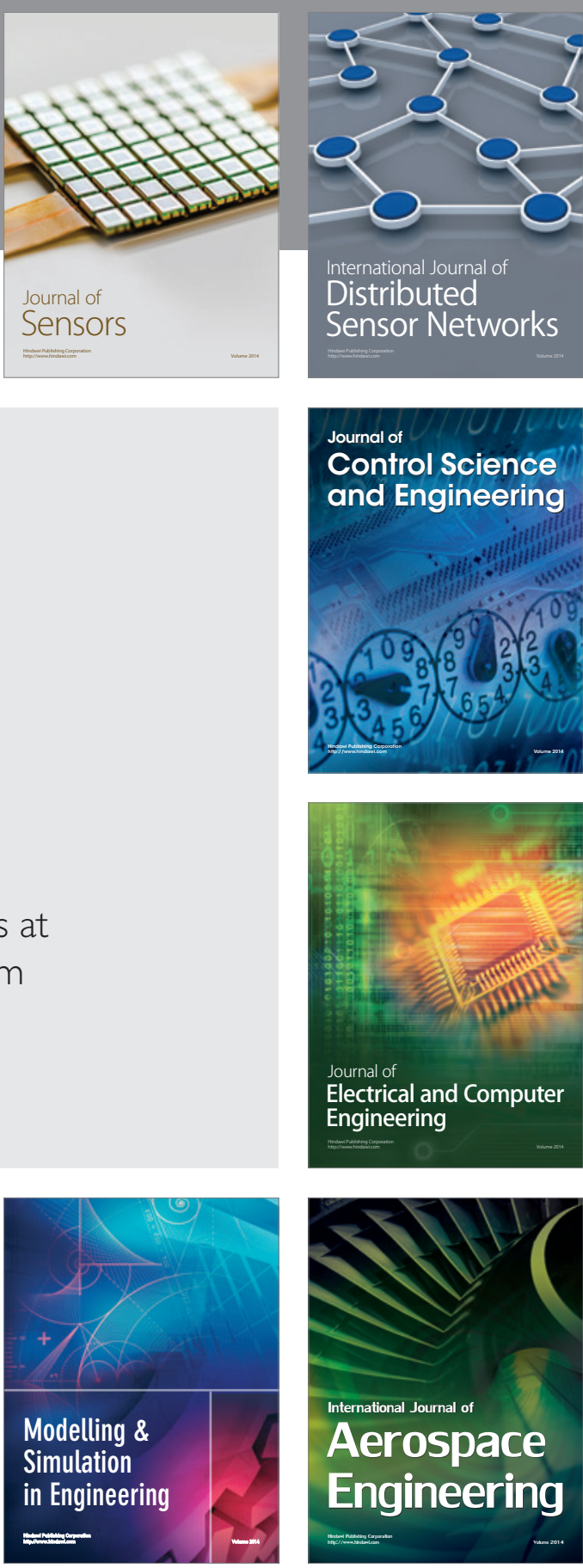

International Journal of

Distributed

Sensor Networks

Journal of

Control Science

and Engineering
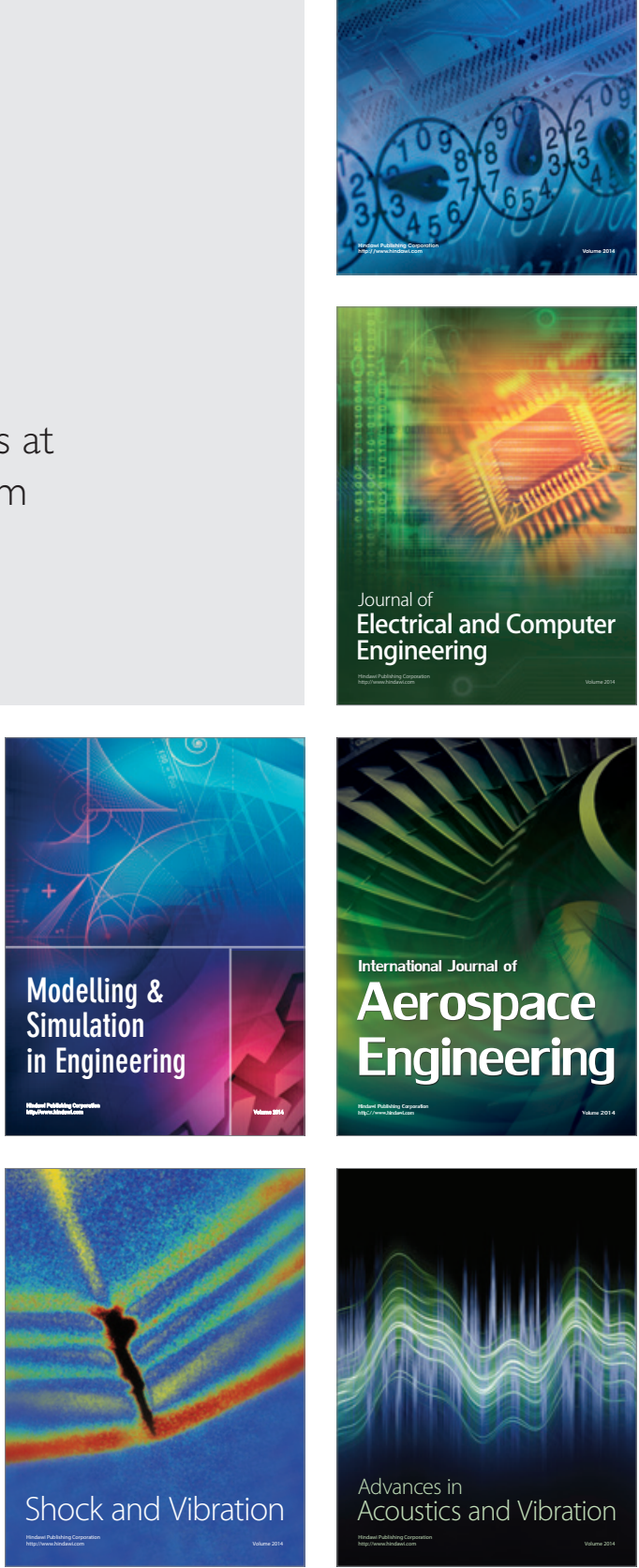